\title{
The role of the magnetic field in the massive star-forming region $\mathrm{W} 75 \mathrm{~N}$
}

\author{
Gabriele Surcis ${ }^{*+\not t}$ \\ Argelander-Institut für Astronomie, Universität Bonn, Germany \\ E-mail: gsurcis@astro.uni-bonn.de
}

\section{Wouter Vlemmings}

Argelander-Institut für Astronomie, Universität Bonn, Germany

E-mail: wouter@astro.uni-bonn.de

\begin{abstract}
A number of different formation scenarios of high-mass have been proposed. However, due to the difficulties observing magnetic fields during the protostellar phase of high-mass star-formation, several questions remain unanswered, e.g., how do magnetic fields influence the formation and dynamics of disks and outflows? Most current information on magnetic fields close to high-mass protostars comes from polarized maser emissions, which allow us to investigate the magnetic field on small scales by using very long baseline interferometry such as EVN and VLBA. Recently, by using maser observations at $6.7 \mathrm{GHz}$ (methanol) and $22 \mathrm{GHz}$ (water), we were able to detect the magnetic field in the massive star-forming region $\mathrm{W} 75 \mathrm{~N}$. In particular we detected two different magnetic fields around the two radio sources VLA 1 and VLA 2, which are at two different evolutionary stages.
\end{abstract}

ISKAF2010 Science Meeting - ISKAF2010

June 10-14, 2010

Assen, the Netherlands

\footnotetext{
${ }^{*}$ Speaker.

$\dagger$ This research is supported by the Deutsche Forschungsgemeinshaft (DFG) through the Emmy Noether research grant VL 61/3-1.

${ }^{\ddagger}$ Member of the International Max Planck Research School (IMPRS) for Astronomy and Astropysics at the Universities of Bonn and Cologne.
} 


\section{Introduction}

In one of the proposed massive star-formation scenarios, core accretion [1], massive stars form through gravitational collapse, which involves disk-assisted accretion to overcome radiation pressure. This scenario is similar to the favoured picture of low-mass star formation [2]. Although magnetic fields are attributed an important role in the formation of low-mass stars, their role during the protostellar phase of high-mass star-formation is still under debate. In particular, it is still unclear how magnetic fields influence the formation and dynamics of disks and outflows. The best probes of the magnetic fields on the smallest scales in the high-mass star-forming environment are maser lines. In fact, the bright and narrow spectral line emission of masers is ideal for detecting, with polarimetric interferometry, both Zeeman-splitting and the orientation of the magnetic field. So far, the main maser species used for this purpose are $\mathrm{H}_{2} \mathrm{O}$ and $\mathrm{OH}$ masers and only recently methanol $\left(\mathrm{CH}_{3} \mathrm{OH}\right)$ maser linear polarization observations have been made (e.g., [3]; [4]). Both $\mathrm{H}_{2} \mathrm{O}$ and $\mathrm{CH}_{3} \mathrm{OH}$ are non-paramagnetic molecules, and thus any linear polarization is a few percent $\left(P_{1}<8 \%\right)$, while circular polarization is well below $1 \%$.

$\mathrm{W} 75 \mathrm{~N}$ is an active high-mass star-forming region in the molecular complex DR21-W75 [5] at a distance of $2 \mathrm{kpc}$. At the resolution of $\sim 1^{\prime \prime} .5$ three regions were identified [6]: W75N(A), W75N (B) and W75N(C). In 1997, with $\sim 0^{\prime \prime}$.1 resolution, three radio sources in W75(B) were detected [7]: VLA 1, VLA 2 and VLA 3. These three sources seems to be at different evolutionary stages [7], in particular VLA 1 is the "oldest" source of this region and VLA 3 the "youngest" one. In this evolutionary scenario VLA 2 is in an intermediate stage. A large scale high-velocity outflow, with an extension greater than $3 \mathrm{pc}$ and a total molecular mass greater than $255 \mathrm{M}_{\odot}$, was also detected from W75N (B) (e.g., [8]). A multi-outflow scenario was also proposed, where VLA 2 may drive the large-scale outflow, and VLA 1 and VLA 3 are the center of other two small flows [8]. However, so far, it has been impossible to determine which is the main powering source of the $3 \mathrm{pc}$ outflow. Several maser species were detected in W75N(B) (hereafter simply W75N): $\mathrm{H}_{2} \mathrm{O}$ (e.g., [7]), $\mathrm{OH}$ (e.g., [9]), and $\mathrm{CH}_{3} \mathrm{OH}$ [10]. Most of those were detected around the two $\mathrm{H}$ II regions VLA 1, here $\mathrm{H}_{2} \mathrm{O}$ and $\mathrm{CH}_{3} \mathrm{OH}$ masers are located along a linear structure parallel to its radio jet, and VLA2, here $\mathrm{H}_{2} \mathrm{O}$ masers showed a circular distribution. $\mathrm{No} \mathrm{CH}_{3} \mathrm{OH}$ masers are associated to VLA 2 (e.g., [10] and references therein). The $\mathrm{OH}$ masers are located between VLA 1 and VLA 2 (e.g., [11]) and on a ring structure around the three radio sources [12].

In this proceeding we give a summary of the results about the orientation and the strength of the magnetic fields around VLA 1 and VLA 2 obtained by studying the $\mathrm{CH}_{3} \mathrm{OH}(6.7 \mathrm{GHz}$; [13]) and $\mathrm{H}_{2} \mathrm{O}$ (22 GHz, [14]) maser emissions by using full polarization observations made with the European VLBI Network (EVN) and the Very Large Baseline Array (VLBA), respectively.

\section{Methanol masers}

W75N was observed in full polarization spectral mode at $6668.518 \mathrm{MHz}$ with 8 of the EVN antennas on June 14th 2008. The bandwidth was $2 \mathrm{MHz}$, providing a velocity coverage of $\sim$ $100 \mathrm{~km} \mathrm{~s}^{-1}$. The resulting beam-size was $8.75 \times 6.45$ mas. The data were correlated twice. First, with modest spectral resolution (512 channels; channel spacing $\sim 0.2 \mathrm{~km} \mathrm{~s}^{-1}$ ), which enabled us to generate all 4 polarization combinations (RR, LL, RL, LR). The second correlation with high 


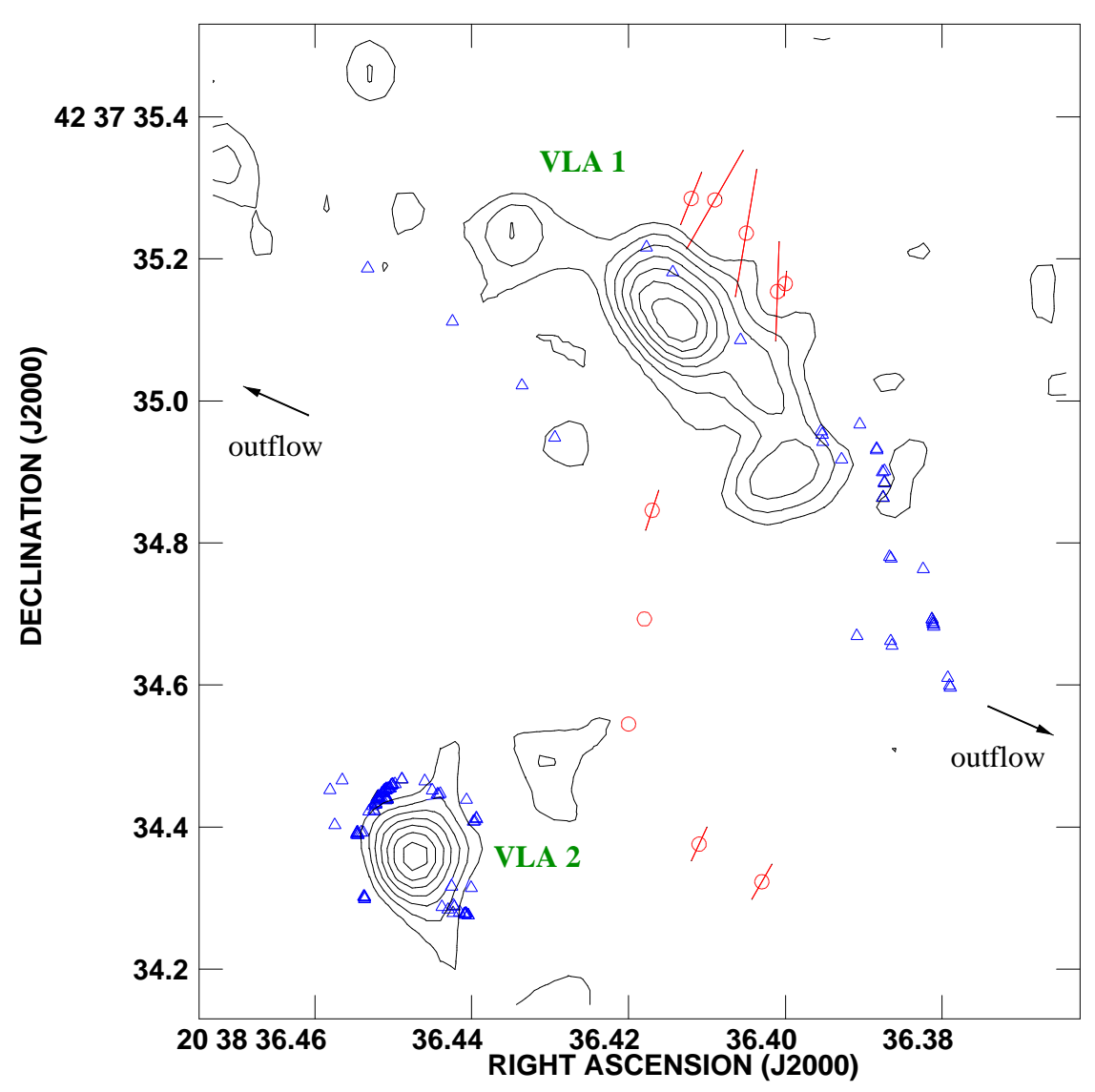

Figure 1: Positions of $\mathrm{CH}_{3} \mathrm{OH}$ (red circles) and $\mathrm{H}_{2} \mathrm{O}$ (blue triangles) masers superimposed on the $1.3 \mathrm{~cm}$ continuum contour map of VLA 1 and VLA 2 [7]. At a distance of $2 \mathrm{kpc} 200$ mas $\approx 400$ AU. The linear polarization vectors of $\mathrm{CH}_{3} \mathrm{OH}$ masers are also reported (40 mas correspond to a linear polarization fraction of $1 \%$ ). Consider that in this case the magnetic field is perpendicular to the linear polarization vectors. The two arrows indicate the direction of the bipolar outflow $\left(66^{\circ}\right)$.

spectral resolution (1024 channels; channel spacing $\sim 0.1 \mathrm{~km} \mathrm{~s}^{-1}$ ) only provided the circular polarization combinations (LL, RR). The details of the calibration are described further in [13].

We have detected $10 \mathrm{CH}_{3} \mathrm{OH}$ maser features, with peak flux density (I) between 2 and 95 Jy beam $^{-1}$ and velocities $3 \mathrm{~km} \mathrm{~s}^{-1}<V_{\mathrm{lrs}}<10 \mathrm{~km} \mathrm{~s}^{-1}$, the 4 southern features were undetected previously [10] (Fig.1). Linear and circular polarization were detected in 8 and 3 of 10 maser features, respectively. The highest fractional linear polarization $\left(P_{1} \sim 2-5 \%\right)$ was detected in the northern features. The linear polarization fraction of the southern masers is slightly lower $\left(P_{1} \sim 1-2 \%\right)$. No dependence of $P_{1}$ on maser brightness is found. The weighted polarization angle of methanol maser vectors is $\langle\chi\rangle=-17^{\circ} \pm 10^{\circ}$. From the theory of polarized masers [15] we found that the linear polarization vectors are perpendicular to the direction of the magnetic field. Consequently, in our case the magnetic field is oriented NE-SW with a position angle of about $73^{\circ} \pm 10^{\circ}$. The magnetic field derived from the $\mathrm{CH}_{3} \mathrm{OH}$ emissions is thus almost perfectly aligned with the large-scale molecular bipolar outflow, which is thought to be oriented close to the plane of the sky at a position angle of $66^{\circ}$. The magnetic field strength, which is derived using a Zeeman-splitting coefficient of $0.049 \mathrm{~km} \mathrm{~s}^{-1} \mathrm{G}^{-1}[3]$ and assuming the angle between the magnetic field and the line of sight to be 
$\theta \sim 70^{\circ}$, is about $50 \mathrm{mG}$. This value is in agreement with the value obtained studying the $\mathrm{OH}$ flare, which is located between the two sources. During this $\mathrm{OH}$ flare a magnetic field strength of $70 \mathrm{mG}$ was measured [11]. Comparing the magnetic field pressure and the dynamics pressure of the gas we can conclude that the magnetic field must play a dominant role in the dynamics of the W75N star-forming region.

\section{Water masers}

We observed W75N in the $6_{16}-5_{23}$ transition of $\mathrm{H}_{2} \mathrm{O}$ (rest frequency: $22.23508 \mathrm{GHz}$ ) with the NRAO VLBA on November $21^{\text {st }} 2005$. The observations were made in full polarization spectral mode using 4 overlapped baseband filters of $1 \mathrm{MHz}$ in order to cover a total velocity range of $\approx 50 \mathrm{~km} \mathrm{~s}^{-1}$. Even in this case two correlations were performed. One with 128 channels (RR, LL, RL, LR) with a spectral resolution of $7.8 \mathrm{kHz}\left(0.1 \mathrm{~km} \mathrm{~s}^{-1}\right)$ and the other one with 512 channels (LL, RR) with a spectral resolution of $1,96 \mathrm{kHz}\left(0.027 \mathrm{~km} \mathrm{~s}^{-1}\right)$. The resulting beam-size was $1.20 \times 0.57$ mas. The details of the calibration and of the analysis are described further in [14].

VLA 1. We have detected $36 \mathrm{H}_{2} \mathrm{O}$ maser features, with $0.4 \mathrm{Jybeam}^{-1}<\mathrm{I}<158 \mathrm{Jybeam}^{-1}$ and $8 \mathrm{~km} \mathrm{~s}^{-1}<V_{\text {lrs }}<24 \mathrm{~km} \mathrm{~s}^{-1}$ around VLA 1, which can be divided in three groups: A, B and C (Fig.2). The highest fractional linear polarization was detected in group B $\left(P_{1} \sim 12-26 \%\right)$, which indicates a saturation level of those $\mathrm{H}_{2} \mathrm{O}$ masers. This can also be see in the scatter of their linear polarization vectors (Fig.2) which gives a weighted polarization angles of $\left\langle\chi_{1 \mathrm{~B}}\right\rangle \approx-56^{\circ}$. The weighted polarization angles for the other two groups are $\left\langle\chi_{1 \mathrm{~A}}\right\rangle \approx-19^{\circ}$ and $\left\langle\chi_{1 \mathrm{C}}\right\rangle \approx-17^{\circ}$, and in these regions the magnetic field is perpendicular to the linear polarization vectors. Consequently the magnetic field orientation around VLA 1 is close to $70^{\circ}$ in agreement with the results obtained in $\$ 2$. Hence, the magnetic field is parallel to the large-scale molecular bipolar outflow. The $\mathrm{H}_{2} \mathrm{O}$ maser around VLA 1 show a $B_{\|}$strength range from -400 to $600 \mathrm{mG}$ indicating a twisted magnetic field. Since $\langle\theta\rangle_{\mathrm{VLA} 1}=83^{\circ}$ we get $\left\langle\left|B_{\mathrm{VLA} 1}\right|\right\rangle \approx 665 \mathrm{mG}$. The strength of the magnetic field depends on the density of the maser $\left(B \propto n_{\mathrm{H}_{2}}^{0.5}\right.$ ), hence the different magnetic field strength determined by using two different maser species, i.e. $\sim 50 \mathrm{mG}$ using $\mathrm{CH}_{3} \mathrm{OH}$ and $\sim 700 \mathrm{mG}$ using $\mathrm{H}_{2} \mathrm{O}$, implies that the $\mathrm{H}_{2} \mathrm{O}$ masers trace gas $\sim 200$ times more dense than the $\mathrm{CH}_{3} \mathrm{OH}$ masers.

VLA 2. We have detected $88 \mathrm{H}_{2} \mathrm{O}$ maser features, with $0.7 \mathrm{Jybeam}^{-1}<\mathrm{I}<198 \mathrm{Jybeam}^{-1}$ and $-8 \mathrm{~km} \mathrm{~s}^{-1}<V_{\text {lrs }}<19 \mathrm{~km} \mathrm{~s}^{-1}$. The $\mathrm{H}_{2} \mathrm{O}$ masers are distributed elliptically around its $1.3 \mathrm{~cm}$ continuum peak. In Fig. 2 (right panel) we reported an ellipse obtained by fitting the positions of the water masers. The masers around VLA 2 have a linear polarization fraction $P_{1}<6 \%$, and their linear polarization vectors are well aligned with the ellipse, even if some of them show the famous $90^{\circ}$-flip. The weighted linear polarization angles of the water masers is $\left\langle\chi_{2}\right\rangle \approx-75^{\circ}$. Consequently the magnetic field orientation is about $15^{\circ}$ that indicates a misalignment with the large-scale molecular bipolar outflow and the magnetic field of VLA 1. From the circular polarization emission the magnetic field strength along the line of sight is $-200 \mathrm{mG}<\mathrm{B}_{\|}<1000 \mathrm{mG}$, in this case the negative and positive strengths of the magnetic fields are well distributed (see Fig.2). Now we have $\left\langle\left|B_{\mathrm{VLA} 2}\right|\right\rangle \approx 1224 \mathrm{mG}\left(\langle\theta\rangle_{\mathrm{VLA} 2}=85^{\circ}\right)$. 

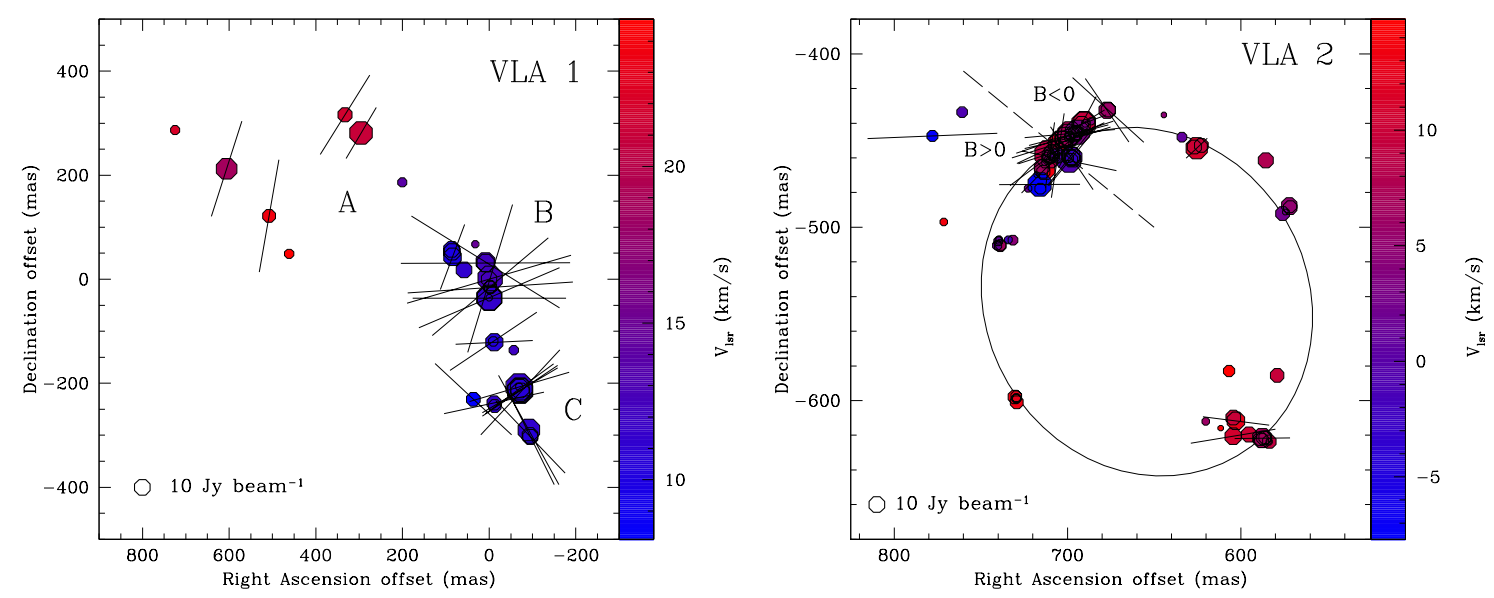

Figure 2: A close up view of the $\mathrm{H}_{2} \mathrm{O}$ masers around VLA 1 (left panel) and VLA 2 (right panel). The reference point is $\alpha_{2000}=20^{\mathrm{h}} 38^{\mathrm{m}} 36^{\mathrm{s}} .4782449$ and $\delta_{2000}=+42^{\circ} 37^{\prime} 34^{\prime \prime} .346438$. The octagonal symbols are scaled logarithmically according to their peak flux density. the linear polarization vectors are scaled logarithmically according to polarization fraction. The maser velocity is indicated by color. Right panel: an ellipse is also draw, which is the result of the best fit of the water masers detected in the present work. The dashed line divides the region with positive and negative magnetic field strengths.

\section{Summary}

We have demonstrated the power of methanol maser polarization observations in deducing the strength and structure of the magnetic field around massive protostars. The magnetic field around VLA 1 is parallel to the large-scale molecular bipolar outflow at small scales, indicating VLA 1 as its powering source. The magnetic field around VLA 2 is instead not related with the outflow, suggesting a different evolutionary stage between the two sources, as reported in [7].

\section{References}

[1] C.F. McKee \& J. Tan, The Formation of Massive Stars from Turbulent Cores, ApJ 585 (2003) 850.

[2] F.H. Shu, J. Najita, E.C. Ostriker et al., Magnetocentrifugally Driven Flows from Young Stars and Disks. V. Asymptotic Collimation into Jets, ApJ 455 (1995) L115.

[3] W.H.T. Vlemmings, L. Harvey-Smith and R.J. Cohen, Methanol maser polarization in W3(OH), MNRAS 371 (2006) L26.

[4] R. Dodson, First VLBI observations of methanol maser polarisation, in G339.88-1.26, A\&A 480 (2008) 767.

[5] J.R. Dickel, H.R. Dickel and W.J. Wilson, The detailed structure of CO in molecular cloud complexes. II - The W75-DR 21 region, ApJ 223 (1978) 840.

[6] A.D. Haschick, M.J. Reid, B.F. Burke et al., VLBI aperture synthesis observations of the OH maser source W75N, ApJ 244 (1981) 76.

[7] J.M. Torrelles, J.F. Gómez, L.F. Rodríguez et al., A Radio Jet-H $2 O$ Maser System in W75N(B) at a 200 AU Scale: Exploring the Evolutionary Stages of Young Stellar Objects, ApJ 489 (1997) 744. 
[8] D.S. Shepherd, L. Testi and D.P. Stark, Clustered Star Formation in W75 N, ApJ 584 (2003) 882.

[9] V.I. Slysh, V. Migenes, I.E. Val'tts et al., Total Linear Polarization in the OH Maser W75 N: VLBA Polarization Structure, ApJ 564 (2002) 317.

[10] V. Minier, R.S. Booth and J.E. Conway, VLBI observations of 6.7 and $12.2 \mathrm{GHz}$ methanol masers toward high mass star-forming regions. I. Observational results: protostellar disks or outflows?, A\&A 362 (2000) 1093.

[11] V.I. Slysh, A.V. Alakov and V. Migenes, Evolution of the spectrum and VLBI structure of W75N during the huge OH maser flare in 2003-2007, MNRAS 404 (2010) 1128.

[12] B. Hutawarakorn, R.J. Cohen and G.C. Brebner, OH masers and magnetic fields in the bipolar outflow source W75N, MNRAS 330 (2002) 349.

[13] G. Surcis, W.H.T. Vlemmings, R. Dodson and H.J. van Langevelde, Methanol masers probing the ordered magnetic field of W75N, A\&A $\mathbf{5 0 6}$ (2009) 757.

[14] G. Surcis, W.H.T. Vlemmings, S. Curiel et al., The structure of the magnetic field in the massive star-forming region $W 75 \mathrm{~N}$, in preparation.

[15] P. Goldreich, D.A. Keeley and J.Y. Kwan, Astrophysical Masers. 11. Polarization Properties, ApJ 179 (1973) 111. 